\title{
Mechanism of complex fracture creation in hydraulic fracturing
}

\author{
Masaya NAGASO ${ }^{1}$, Hitoshi MIKADA ${ }^{2}$ and Junichi TAKEKAWA ${ }^{2}$ \\ ${ }^{1}$ Dept. of Civil and Earth Res. Eng., Kyoto University (Now at Geothermal Engineering Co., Ltd.) \\ ${ }^{2}$ Dept. of Civil and Earth Res. Eng., Kyoto University
}

\begin{abstract}
Hydraulic fracturing is an essential technique for the development of unconventional oil reservoirs. If fracture network formation is evaluated before a real practice of hydraulic fracturing, the permeability of the rock could be optimized after the fracturing. Since a lot of factors are involved in fracture complexity, the mechanism of fracture network formation is not fully revealed. Although the strength heterogeneities of rock mass is known to be one of the factors, strength heterogeneities is rarely taken into consideration because of less understanding of the influence on complex fracture creation. We perform a series of numerical simulation using the discrete element method and investigate the mechanism of fracture network formation, focusing on the strength heterogeneities and brittleness, which is often used as an index of fracture network formation. In heterogeneous models, complex fracture is formed by micro cracks generated around the tip of main fracture and pores with specific shape. On the other hand, in a model with high brittleness, a lot of branches are created by shear failure with main fracture propagation. These results indicate that the mechanism of complex fracture formation due to strength heterogeneities is completely different from that due to brittleness, and that the effect of the strength heterogeneities of rock should be considered as a key factor of the complication of fracture networks.
\end{abstract}

\section{Introduction}

Hydraulic fracturing is a method to improve the permeability of the rock in the development of unconventional reservoirs. Complex fracture network formed by hydraulic fracturing enhances the permeability of the rock significantly. It is known that the formation of fracture network determines the production of resources and therefore, accurate evaluation for fracture network formation is necessary. Fracture shape induced by fracking depends on many geological factors such as stress field, brittleness, and existence of pre-existing fractures ${ }^{1)}$. Understanding these geological factors and choosing appropriate flow rate, viscosity, and total mass of injection fluid, ideal fracture network could be created. However, the mechanism of complex fracture creation due to the geological factors is complicated, and accurate evaluation for fracture network formation is still significant challenge. Although strength heterogeneities is also known to have great influence on complex fracture creation ${ }^{2)}$, strength heterogeneities of rock is rarely taken into consideration in evaluation of fracture network formation in hydraulic fracturing. This is mainly because the mechanism of complex fracture formation due to strength heterogeneities is not fully revealed. Therefore, the evaluation of fracture network formation could be more accurate by revealing the relation between strength heterogeneities and fracture complexity.

In the present study, we conduct a series of numerical experiments of hydraulic fracturing using discrete element method (DEM) and investigate the mechanism of complex fracture creation due to strength heterogeneities. We focus not only on the effect of strength heterogeneities but also on that of brittleness, and compare these effects.

\section{Discrete element method}

We create an original code of the 2D DEM to simulate hydraulic fracturing. We will give a brief explanation in this paper due to limitations of space. Details can be found in a reference ${ }^{3)}$.

The total force acting on each particle is comprised of a force arising from particle-particle overlap and a force arising from parallel bond. The increments of normal force $\bar{f}_{n}$, tangential force $\bar{f}_{s}$, and moment $\bar{M}$ carried by the parallel bond can also be calculated from relative motion of the bonded particles, and given as

$$
\overline{f_{n}}=\overline{k_{n}}\left(d n_{j}-d n_{i}\right)
$$




$$
\begin{gathered}
\overline{f_{s}}=\overline{k_{s}}\left[d s_{j}-d s_{i}-\left(r_{i} d \theta_{i}+r_{j} d \theta_{j}\right)\right] \\
\bar{M}=\overline{k_{\theta}}\left(d \theta_{j}-d \theta_{i}\right)
\end{gathered}
$$

where $\overline{k_{n}}, \overline{k_{s}}$, and $\overline{k_{\theta}}$ are the stiffness of normal, shear, and rotational spring of the parallel bond, and $d n, d s$, and $d \theta$ are normal and shear displacement and rotation of a particle. $r$ is radius of a particle. These stiffnesses are given as the following equation by the beam theory.

$$
\begin{aligned}
& \overline{k_{n}}=\frac{E D}{L} \\
& \overline{k_{s}}=\alpha \overline{k_{n}} \\
& \overline{k_{\theta}}=\frac{E I}{L}
\end{aligned}
$$

Where $L, D, E, \alpha$, and $I$ are length, diameter, Young's modulus, stiffness ratio, and inertia of the parallel bond. Normal stress $\sigma$ and shear stress $\tau$ act on contact point of the bonded particles and are given as follows.

$$
\begin{gathered}
\sigma=\frac{\overline{f_{n}}}{\bar{D}} \\
\tau=\frac{\overline{f_{s}}}{\bar{D}}
\end{gathered}
$$

When $\sigma$ exceeds the tensile strength $\sigma_{c}$ or $\tau$ exceeds the shear strength $\tau_{c}$, the bond breaks. Criterions for the bond break can be written as follows.

$$
\begin{gathered}
\sigma \leq-\sigma_{c} \\
|\tau| \geq \tau_{c}
\end{gathered}
$$

When each bond breaks, a micro crack is generated at the contact point of the particles.

To simulate fluid flow, the fluid flow algorithm is introduced into the DEM program. In the fluid flow algorithm, pores are assumed to be in the center of the enclosed domain, and flow channels connecting pores are assumed to be at the contact points of particles. Since the fluid flow in the flow channels is assumed to be the laminar flow, the flow rate is calculated by using the Poiseuille equation and is given as

$$
Q=\frac{w^{3}}{12 \mu} \frac{\Delta P}{L_{p}}
$$

where $w$ is aperture, $L_{p}$ is length of the flow channel, $\Delta P$ is pressure difference of two neighboring pores, and $\mu$ is viscosity of the fluid. Each pore accumulates the fluid pressure and the pressure increment at each pore is given as

$$
\Delta P=\frac{K_{f}}{V_{r}}\left(\sum Q d t-d V_{r}\right)
$$

where $K_{f}$ is fluid bulk modulus, $V_{r}$ is current pore volume, $\sum Q$ is total flow rate from the surrounding channels, $d V_{r}$ is change of volume of the pore.

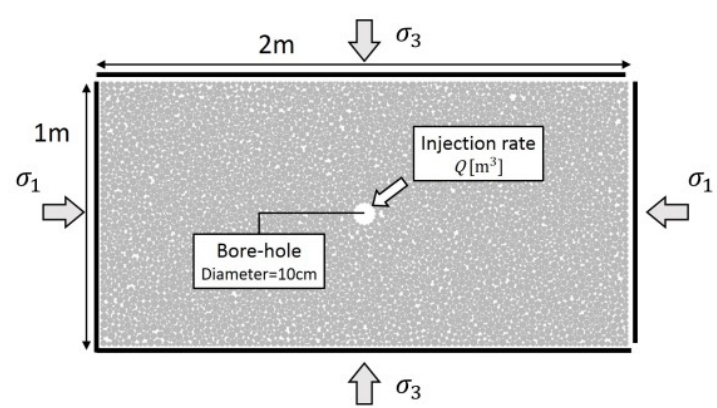

Figure 1 Numerical model for hydraulic fracturing

Table 1 Model parameters in section 3

\begin{tabular}{|lc|}
\hline Model parameter & \\
\hline Young modulus[GPa] & 70.0 \\
Poison's ratio & 0.3 \\
UCS [MPa] & 199.9 \\
UTS [MPa] & 10.0 \\
Permeability [m ${ }^{2}$ ] & $1.0 \times 10^{-17}$ \\
\hline
\end{tabular}

Fluid pressure accumulated in the pore and shear stress caused by fluid flow is acting on particles. Pore pressure is acting on the particle surface and is given as

$$
f_{d}=\int_{-\beta}^{\beta} P \cos \theta r d \theta
$$

where $\beta$ is corner half-angle of a domain. To consider partially saturated condition, saturation in each pore is introduced (Shimizu et al., 2011). The saturation is given as

$$
S_{t}=\frac{V_{f}}{V_{d} \varphi}
$$

where $V_{d}$ and $V_{f}$ is volume of pore and fluid in the pore, and $\varphi$ is porosity of the model. When $S_{t}>1$, the pore is saturated with fluid and has fluid pressure. On the other hand, when $S_{t}<1$, the pore is not saturated and the fluid pressure is zero.

\section{Complicated fracture due to strength heterogeneities}

Numerical model used in hydraulic fracturing simulation is shown in Fig. 1. Size of the model is 1 $[\mathrm{m}]$ in length and $2[\mathrm{~m}]$ in width. At the center of the model, a bore-hole is set and its diameter is 10 [cm]. $10[\mathrm{MPa}]$ of compression stress is applied to longitudinal wall and $5[\mathrm{MPa}]$ to lateral wall by adjusting right and top wall. During the simulation, fluid is injected with fixed flow rate and fluid pressure is applied on the bore-hole wall. Injection fluid is assumed to be water and its viscosity is the same as that of water, $0.001[\mathrm{mPa} s]$. Table 1 shows 


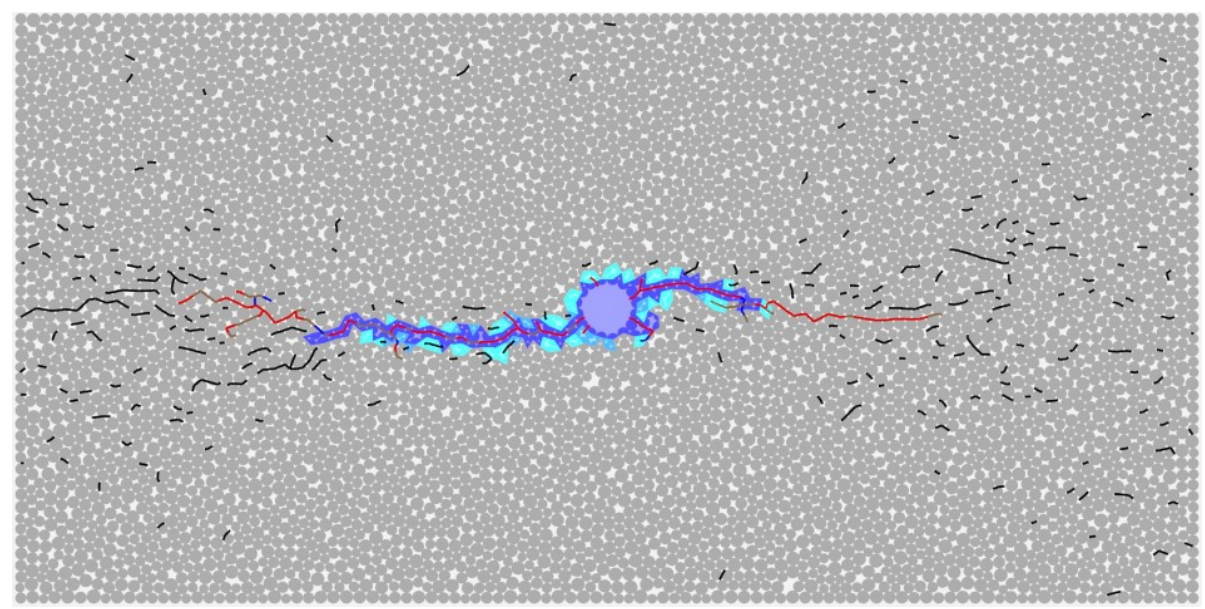

(a) Heterogeneous model

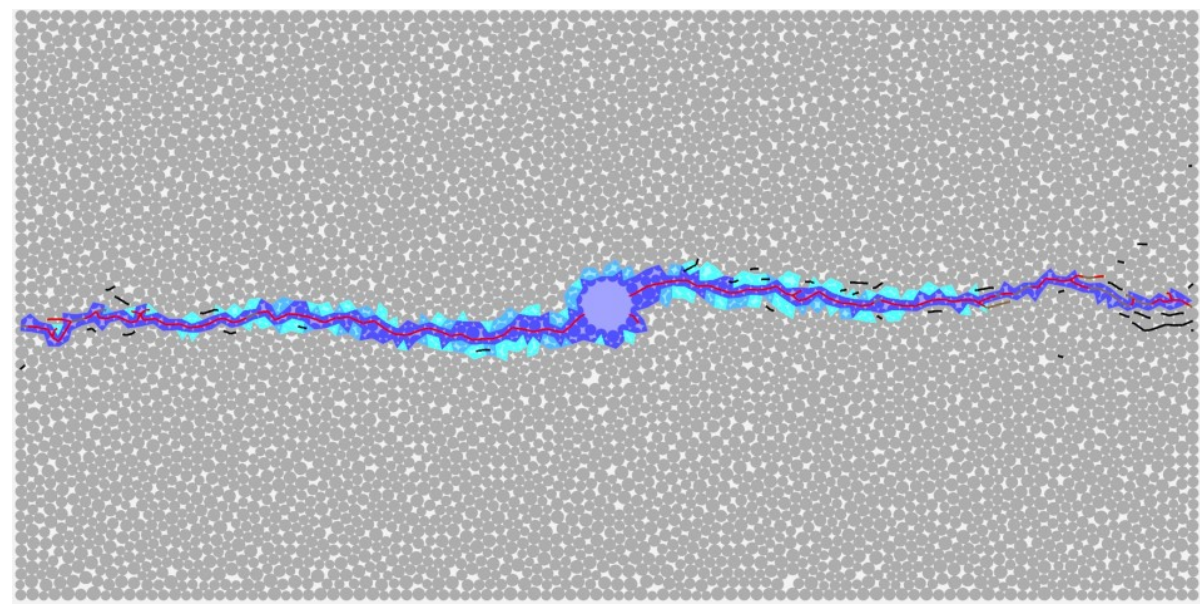

(b) Homogeneous model

Figure 2 Fracture distribution and fluid infiltration as results of numerical simulation

model parameters set by calibration so that numerical model simulates Lac du Bonnet granite ${ }^{3)}$. To reveal the influence of strength heterogeneities on the results of hydraulic fracturing, we prepare 2 numerical models with different strength heterogeneities. Bond strength of both models is distributed by Weibull distribution. Weibull coefficient, which is a shape parameter of the distribution is set to 1.5 for heterogeneous model and 5.0 for homogeneous model.

As a result of numerical simulation, complex fractures are created in heterogeneous model and simple fractures are formed in homogeneous model (Fig.2). Red and blue parts of lines indicate fractures created by tensile and shear breaks, respectively. Black lines indicate micro cracks. Brown parts are created by the connection of main fracture to micro cracks. Blue area indicates saturated area with injected fluid. A lot of branches are created in heterogeneous model mainly by scattered micro cracks and pores with specific shape. In heterogeneous model, a lot of micro cracks are generated around the tip of main fractures because heterogeneous model includes many weak bonds. These micro cracks and the main fracture interact with each other, and then many branches are formed along the main fracture. On the other hand, in homogeneous model, few micro cracks are generated and newly induced fracture is created mainly by opening main fracture as injected fluid infiltrates.

Pores with specific shape also influences complexity of fractures. DEM model with random arrangement of particles includes a lot of pores with specific shape, because shape of each pore is determined by particle arrangement. Pores which are long perpendicularly to the direction has especially great influence on branches formation. Fluid pressure acting in such pores induces tensile stress parallel to the main fracture, and a new fracture is generated at the tip of such pores. Both newly induced fracture and main fracture propagate perpendicularly, and a branch is formed. However, branches are not always created at the long pores, especially in homogeneous model. This means that not only long pores but also weak bonds around the pores are necessary to create branches. In this way, 
Table 2 Model parameters in section 4

\begin{tabular}{|c|c|c|}
\hline Model parameter & $\mathrm{B} 20$ & BI60 \\
\hline Young modulus[GPa] & 10.0 & 55.0 \\
\hline Poison's ratio & 0.2 & 0.2 \\
\hline UCS [MPa] & & 21.4 \\
\hline UTS [MPa] & & 3.8 \\
\hline Permeability $\left[\mathrm{m}^{2}\right]$ & \multirow{2}{*}{\multicolumn{2}{|c|}{$218^{1.0 \times 10^{-19}}$}} \\
\hline BI [\%] & & \\
\hline
\end{tabular}

in heterogeneous model, branches are readily formed at the long pores and complex fractures are induced.

\section{Complex fracture due to brittleness}

Numerical experiments are conducted using numerical models with different brittleness to compare the mechanism of complex fracture formation. Brittleness also has significant influence on the creation of complex fracture in hydraulic fracturing ${ }^{4)}$, but the mechanism of complex fracture creation in brittle rock is not fully revealed. Brittleness of the rock is often used in the evaluation of fracture network formation in shale oil development by using Brittleness Index proposed by Rickman et al. $(2008)^{5}$. Brittleness Index (BI) is estimated from Young modulus and Poisson's ratio, and written as the equation below.

$$
\begin{aligned}
B I[\%]=\frac{1}{2}\left(\frac{E-E_{\min }}{E_{\max }-E_{\min }}-v\right. \\
\left.+\frac{v_{\max }-v_{\min }}{v_{\max }}\right)
\end{aligned}
$$

$E$ and $v$ indicate Young modulus and Poisson's ratio, respectively. Subscript of max and min means that the values are the maximum and the minimum in the specific region. Eq. (15) indicates that the higher Young modulus and the lower Poisson's ration, the higher Brittleness Index becomes. In DEM, brittleness index is expressed by setting macroscopic parameters of Young modulus and Poisson's ratio. These macroscopic parameters are determined to simulate the mechanical property of shale by conducting preliminary simulations of uniaxial compression tests and uniaxial tensile tests. The macroscopic parameters of our two numerical models are shown in Table. 2 . We call our numerical model BI20 and BI60, respectively, according to its brittleness index. The shape of numerical model is the same as the model used in section 3 (Fig. 1). Unlike the numerical model in section 3 , all bonds have the same tensile and share strength. We use lower flow rate of $0.002\left[\mathrm{~m}^{3} / \mathrm{s}\right]$ in this section because the strength of numerical model is lower.
As a result of numerical experiments, complex fracture network with many branches is formed in BI60, while simple fracture is created in BI20 (Fig. 3 ). Many branches are formed by shear failure in BI60. These branches extend perpendicularly to the propagating direction of the main fracture. At some tips of the branches, new fractures are generated and extend in the maximum principle stress direction, and several fractures parallel to each other are formed. Some parallel fractures are connected again by shear failure occurring at the tip of parallel fracture. In this way, complex network is created by shear failures in a numerical model with high brittleness. Shear failure occurring in the numerical model with large Young modulus is caused by strong fluid pressure acting on the fracture surface. Fluid pressure in the bore-hole increases with fluid injection, and micro crack is generated on the borehole wall with the same fluid pressure in BI20 and BI60. Injection fluid flows into the micro crack and fluid pressure in the bore-hole decreases in BI20. The cracks extend with infiltration of fluid and induced fluid pressure and further propagation. On the other hand, in BI60, the width of cracks are too small and injection fluid hardly flows into cracks because high Young modulus of BI60 model restricts the deformation of matrix, and cracks are difficult to be opened. Fluid pressure continues to increase even if cracks is generated on the wall of bore-hole. Stronger fluid pressure acting on the fracture surface induces strong shear stress in the vicinity of the main fracture. Due to the strong shear stress, shear failure occurs and new fractures are generated around the main fracture.

\section{Conclusion}

To investigate the influence of strength heterogeneities on the complication mechanism of the fracture network, a series of numerical experiments is conducted. As a result, we reveal that complex fractures are formed by micro cracks scattered around the tip of the main fracture and fluid pressure acting in specific pores with special shape. On the other hand, in high brittleness model, the complex fracture network is formed due to shear failures because high shear stress in the vicinity of the fracture is induced by strong fluid pressure in fractures. This complication mechanism is completely different from that due to strength heterogeneities. These facts indicate that complex fracture network formation in hydraulic fracturing could be estimated more accurately by considering strength heterogeneities of the rock. 


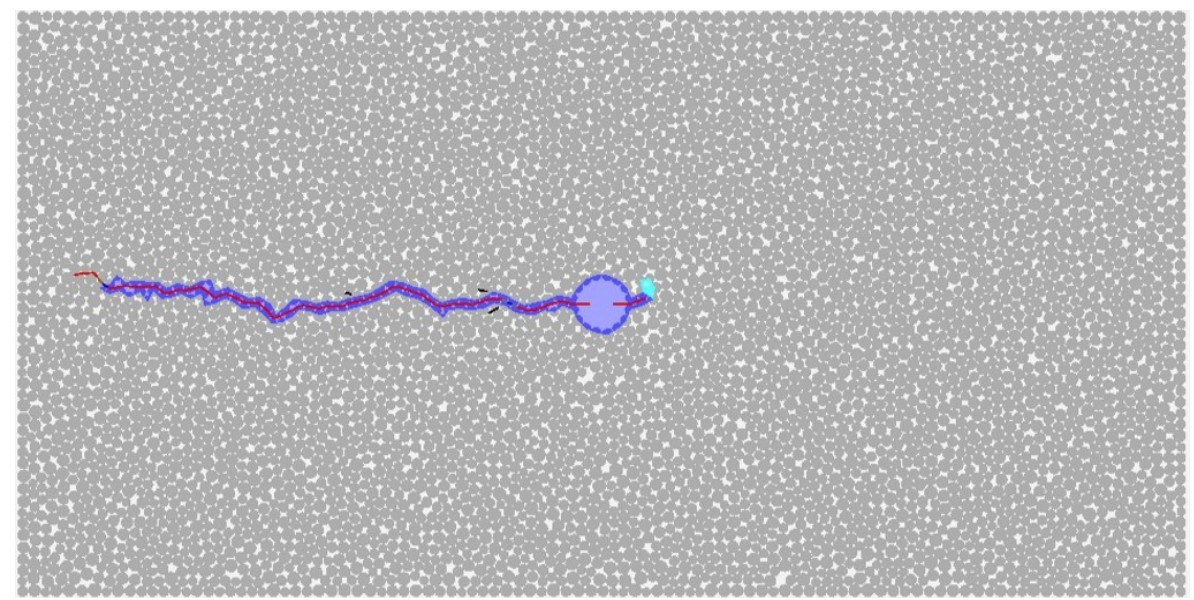

(a) BI 20

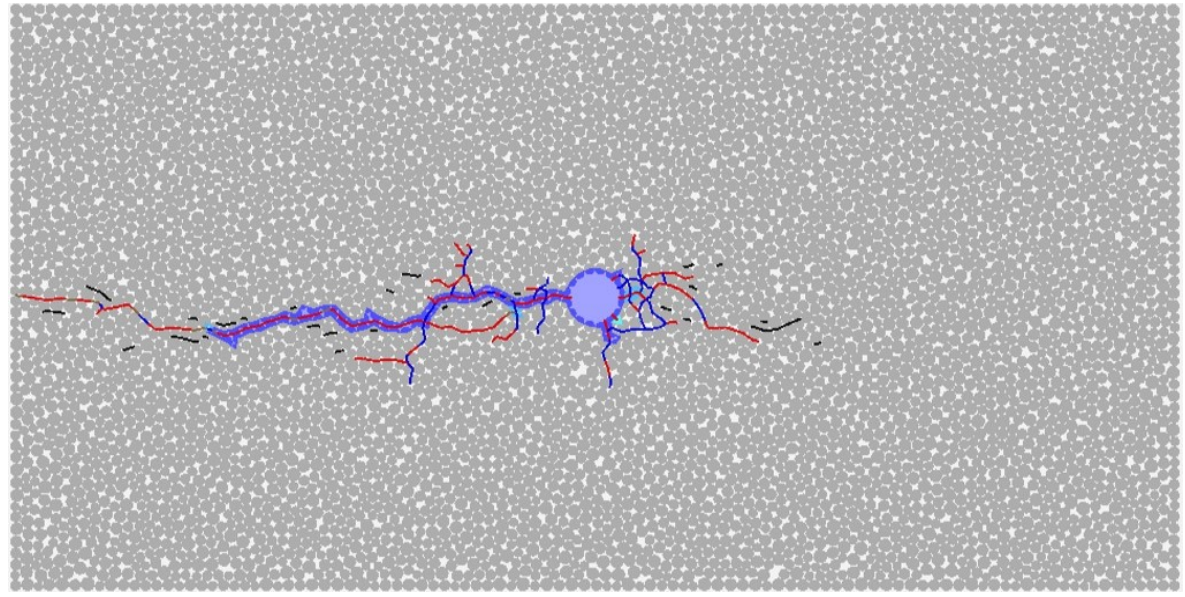

(b) BI 60

Figure 3 Fracture distribution and fluid infiltration as results of numerical simulation

\section{REFERENCES}

1) Ren, L., Zhao, J., \& Hu, Y., 2014, Hydraulic Fracture Extending into Network in Shale: Reviewing Influence Factors and Their Mechanism. The Scientific World Journal, 1-9.

2) Tang, CA., Liu, H., Lee, PKK., Tsui, Y., Tham, L.G., 2000, Numerical studies of the influence of microstructure on rock failure in uniaxial compression - Part I: effect of heterogeneity, Int $J$ Rock Mech Min Sci, 37, 555-569.

3) Potyondy, D.O., Cundall, P.A., 2004, A bondedparticle model for rock. Int J Rock Mech Min Sci, 41, 1329-1364.

4) Hiyama, M., Shimizu, H., Ito, T., Tamagawa, T., \& Tezuka, K, 2013, Distinct element analysis for hydraulic fracturing in shale -Effect of brittleness on the fracture propagation-. 47th US Rock Mechanics / Geomechanics Symposium, San Francisco, CA, USA, 23-26.

5) Rickman, R., Mullen, M., Petre, E., Grieser, B., \& Kundert, D., 2008, A Practical Use of Shale
Petrophysics for Stimulation Design Optimization: All Shale Plays Are Not Clones of the Barnett Shale. Proceedings of 2008 SPE Annual Technical Conference and Exhibition, 21 -24 . 